\title{
Uso de cepas de Pycnoporus sanguineus para la producción de enzimas con potencial uso en la industria de la panificación
}

\author{
Use of Pycnoporus sanguineus strains to the production of enzymes with \\ potential applications on the bread industry
}

\begin{abstract}
Susana Daniela Salinas Sánchez', losvany López-Sandin², Carlos E. Hernández-Luna ${ }^{3}$, Juan Francisco ContrerasCordero ${ }^{3}$, Gerardo Méndez-Zamora', Carlos A. Hernández-Martínez', Guadalupe Gutiérrez-Soto'*.

Universidad Autónoma de Nuevo León, Facultad de Agronomía, Francisco Villa S/N., Col. Ex Hacienda El Canadá, General Escobedo, Nuevo León, México. ZP. 66050.

2 Dpto. de Mecánica Aplicada, Facultad de Ciencias Técnicas, Universidad de Ciego de Ávila, Carretera a Morón, Km 9 1⁄2, Ciego de Ávila, Cuba. ZP. 69450.

3 Universidad Autónoma de Nuevo León, Facultad de Ciencias Biológicas, Pedro de Alba S/N., Ciudad Universitaria, San Nicolás de los Garza, Nuevo León, México. ZP. 66455.
\end{abstract}

\section{RESUMEN}

El uso de enzimas como a-amilasas, xilanasas y glucosidasas es una buena alternativa para obtener una mayor eficiencia en los productos de la industria panificadora, sin dejar de mencionar la lacasa que ha mostrado un potencial prometedor. Así, el objetivo de este estudio fue obtener concentrados enzimáticos de xilanasas, amilasas, celulasas y lacasas a partir de cepas nativas de Pycnoporus sanguineus. Para lo cual, se evaluó el efecto del medio de cultivo sobre la producción de lacasa juntamente con el escrutinio en medio sólido para la detección de xilanasas, amilasas y celulasas. A los concentrados enzimáticos obtenidos se les determinaron las unidades de lacasa y carbohidrolasas. Los títulos más altos de lacasa se presentaron en el medio Tx2. Con respecto a las carbohidrolasas, se detectaron en diferentes niveles de actividad y combinaciones. El concentrado con altos niveles de amilasa y lacasa fue $\mathrm{CH} 116$, mientras que LE90 tuvo la mayor actividad de xilanasa y LE133 de celulasa. La cepa CH116 fue productora de amilasa, celulasa, xilanasa y lacasa, siendo la de mayor potencial en la industria panificadora, mientras que LE90 puede ser utilizada en la industria papelera por su combinación de xilanasas y lacasa.

Palabras clave: basidiomicetos, carbohidrolasas, cepas nativas, lacasa

\section{ABSTRACT}

The use of enzymes such as a-amylases, xylanases and glucosidases is a good alternative to obtain higher efficiency in the bakery industry, not to mention laccase which has shown promising potential. Thus, the objective of this study was to obtain enzyme concentrates of xylanases, amylases, cellulases and laccases from Pycnoporus sanguineus native strains. For which, the effect of culture medium on the laccase production was evaluated together with the solid medium screening of xylanases, amylases and cellulases. Laccase and carbohydrolases units were determined from the enzyme concentrates obtained. The higher laccase titers occurred in the Tx2 medium. Regarding carbohydrolases, they were pre- sent at different levels of activity and combinations. The concentrate with higher titers of amylase and laccase was $\mathrm{CH} 116$, while LE90 had the greater xylanase activity and LE133 of ceIlulase. The $\mathrm{CH} 116$ strain was a producer of amylase, cellulase, xylanase and laccase, being the one with the higher potential in the bakery industry, while LE90 in the paper industry, due to its combination of xylanases and laccase.

Key words: basidiomcietos, carbohydrolases, laccase, native strain.

\section{INTRODUCCIÓN}

A nivel industrial se destaca el uso de las enzimas en todos los sectores (alimentos, piensos, detergentes, textiles, lavandería, farmacéutica, cosmética y química fina), representando el $80 \%$ del mercado mundial de las enzimas. En este contexto, las enzimas alimentarias son las más utilizadas y comprenden la mayor parte del mercado (Miguel et al., 2013). Si bien, las enzimas pueden ser obtenidas a partir de plantas o animales, la mayoría son de fuentes microbianas. Estos organismos tienen ventajas como rápidas tasas de crecimiento, altos rendimientos y son relativamente fáciles de manipular genéticamente (Patel et al., 2017). Por lo que, constantemente se buscan nuevas fuentes de enzimas, capaces de producir altos niveles en medios de bajo costo (Kathirgamanathan et al., 2017).

La industria alimentaria ha desarrollado procesos enzimáticos para su uso en la producción de alimentos, con aplicaciones como la extracción de compuestos bioactivos, mejorar la textura y reducir los riesgos a la seguridad alimentaria (Zhang et al., 2018). Entre otras ventajas, la aplicación de enzimas ha sido elegida ante otros aditivos por la demanda de componentes naturales, además de satisfacer los requerimientos de calidad con sus efectos positivos en procesos como la producción de edulcorantes y panificación (Singh et al., 2019). En particular, la industria de panificación ha usado estas biomoléculas con el propósito de mejorar las características de la masa y el pan, así como aumentar sus atributos nutricionales (Tebben et al., 2018). 
En este sentido, el cumplimiento de los estándares de calidad del pan se obtiene a partir de mezclas complejas de enzimas de diferentes fuentes que actúan sinérgicamente en distintos componentes de la masa. Inicialmente, éstas eran obtenidas a partir de estómagos de terneros, corderos y cabritos. Actualmente, estas biomoléculas se producen industrialmente a partir de microorganismos por su fácil manipulación genética y ventajas en comparación a las enzimas de fuentes animales y vegetales (Singh y Kumar, 2019). Además, el uso de microorganismos en la producción de enzimas a gran escala vuelve el proceso económico y versátil por sus cortos tiempos de producción (Patel et al., 2016).

Entre los microorganismos destacan los hongos por su capacidad de romper biopolímeros como la celulosa o el almidón, convirtiéndolos en una potencial fuente de enzimas degradadoras a nivel industrial. Sin embargo, la producción de enzimas se realiza a partir de la disponibilidad de sustratos que la especie está adaptada a degradar (Drozlowska, 2019). En este sentido, los hongos de la pudrición blanca (WRF) sobresalen entre las especies de basidiomicetos por su capacidad de degradar lignina y celulosa, asociado a la producción de enzimas lignocelulolíticas que les permiten asimilar los compuestos y crecer en diferentes sustratos. Sin embargo, por su baja producción ha sido investigada la inducción de enzimas de WRF para su aplicación a nivel industrial a bajo costo (Vrsanska et al., 2016). Otra alternativa para obtener altos títulos de producción es la prospección de nuevas cepas con alto potencial lignocelulolítico. Uno de los géneros que han destacado en este tipo de estudios es Pycnoporus (Gutiérrez-Soto et al., 2015). Por tal razón, el objetivo de este trabajo fue estudiar el potencial lignocelulolítico de tres cepas nativas de Pycnoporus sanguineus para obtener concentrados enzimáticos con potencial aplicación en la industria de la panificación.

\section{MATERIALES Y MÉTODOS Material biológico}

Las cepas Pycnoporus sangineus LE90, LE133 y CH116 son cepas nativas del estado de Nuevo León, resguardadas en el Laboratorio de Enzimología de la Facultad de Ciencias Biológicas de la Universidad Autónoma de Nuevo León. Las cepas fueron reactivadas en medio de cultivo papa dextrosa agar (PDA, BD Bioxon, México) por 5 días para la preparación del inóculo.

\section{Actividad lacasa}

La actividad lacasa fue determinada midiendo la oxidación de 2,6-dimetoxifenol (DMP) a $468 \mathrm{~nm}\left[\varepsilon_{468}=49,600 \mathrm{M}^{-1}\right.$ $\mathrm{cm}^{-1}$. La mezcla de reacción fue preparada en una solución amortiguadora de acetato de sodio $(200 \mathrm{mM})$, ajustado a $\mathrm{pH}$ 3.5 con $2 \mathrm{mM}$ de DMP (Abadulla et al., 2000). Las actividades enzimáticas se expresaron en unidades $(U)$ definidas como la cantidad de enzima requerida para producir $1 \mu \mathrm{mol}$ de producto por minuto. Las reacciones enzimáticas se llevaron a cabo por triplicado a $25^{\circ} \mathrm{C}$ en un espectrofotómetro UV-Vis 1800 (Shimadzu, Japon).

\section{Carbohidrolasas y azúcares reductores}

Las actividades celulasas, xilanasas y amilasas fueron detectadas determinando la cantidad de azúcares reductores liberados en la reacción siguiendo el método establecido por Miller et al. (1959). Las mezclas de reacción consistieron en $0.5 \mathrm{~mL}$ de la solución amortiguadora citrato de sodio (50 $\mathrm{mM})$, ajustado a $\mathrm{pH}$ 5.0, $0.3 \mathrm{~mL}$ de sustrato $1 \%(\mathrm{w} / \mathrm{v})$ dependiendo de la actividad a determinar (CM-celulosa, número de catálogo C4146; D-xilano, número de catálogo X0502; o almidón, número de catálogo 33615; Sigma-Aldrich Corp. (St. Louis, MO, USA)) y $0.2 \mathrm{~mL}$ de muestra. Las reacciones fueron incubadas por $15 \mathrm{~min}$ a $60^{\circ} \mathrm{C}$. Para la cuantificación azúcares reductores fueron agregados $0.1 \mathrm{~mL}$ de la mezcla de reacción a $0.1 \mathrm{~mL}$ de ácido dinitrosalicílico (DNS) (Miller et al., 1959), fueron hervidas durante $5 \mathrm{~min}$ a $100{ }^{\circ} \mathrm{C}$ y posteriormente sumergidas en un baño con hielo a $-4{ }^{\circ} \mathrm{C}$. Después, para determinar fotométricamente la absorbancia de las muestras se les agregó $1 \mathrm{~mL}$ de agua bidestilada y se procedió a leer a una longitud de onda de $540 \mathrm{~nm}$ en espectrofotómetro (Shimadzu UV-Vis 1800, Japón). Para la cuantificación de las celulasas y amilasas fue utilizada una curva de glucosa en un rango de 0 a $1 \mathrm{mg}$, para las xilanasas fue utilizada una curva de xilano en el mismo rango. Una unidad de enzima (U) fue definida como la cantidad de enzima requerida para liberar $1 \mu \mathrm{mol}$ de glucosa o xilano por minuto. Las reacciones enzimáticas se llevaron a cabo por triplicado a $25^{\circ} \mathrm{C}$.

\section{Cinética de producción de lacasa}

La cinética de producción y el efecto de la composición del medio sobre lacasa fueron realizados en medio Kirk modificado (Pozdnyakova et al., 2006). La composición consistió en $5 \mathrm{~g} \mathrm{l}^{-1}$ de peptona (Bacto peptone, BD BIOXON México), $1 \mathrm{~g} \mathrm{l}^{-1}$ de extracto de levadura (BD BIOXON México), $2 \mathrm{~g} \mathrm{l}^{-1}$ de tartrato de amonio, $1 \mathrm{~g} \mathrm{l}^{-1}$ de $\mathrm{KH}_{2} \mathrm{PO}_{4^{\prime}} 0.5 \mathrm{~g} \mathrm{l}^{-1} \mathrm{de}$ $\mathrm{MgSO}_{4^{\prime}} 0.5 \mathrm{~g} \mathrm{l}^{-1}$ de KCl, $10 \mathrm{~mL} \mathrm{l}^{-1}$ de la solución de elementos DMS 100x. La solución DMS contenía $5 \mathrm{~g} \mathrm{l}^{-1}$ de EDTA, $2 \mathrm{~g} \mathrm{l}^{-1}$ de $\mathrm{FeSO}_{4^{\prime}} 0.1 \mathrm{~g} \mathrm{l}^{-1}$ de $\mathrm{ZnSO}_{4^{\prime}} 0.03 \mathrm{~g} \mathrm{l}^{-1}$ de $\mathrm{MnCl}_{2^{\prime}} 0.3 \mathrm{~g} \mathrm{l}^{-1} \mathrm{de} \mathrm{H}_{2} \mathrm{BO}_{4^{\prime}}$ $0.2 \mathrm{~g} \mathrm{l}^{-1}$ de $\mathrm{CoCl}_{2}, 0.01 \mathrm{~g} \mathrm{l}^{-1}$ de CuSO y $0.03 \mathrm{~g} \mathrm{l}^{-1}$ de $\mathrm{NaMoO}_{4}$. A partir de este fueron establecidas tres condiciones de medio de cultivo, donde lo medio Tx1 fue elaborado con All Bran Flakes ${ }^{\circledR}$ (BF) al $2 \%$ como fuente de carbono. El medio Tx2 además del BF al $2 \%$, fue suplementado con $\mathrm{CuSO}_{4}(350 \mu \mathrm{M})$ como inductor de lacasa. El medio Tx3 contenía glucosa (10 $\left.\mathrm{g} \mathrm{l}^{-1}\right)$ y xilano $\left(20 \mathrm{~g} \mathrm{l}^{-1}\right)$ como fuentes de carbono y CuSO $\mathrm{C}_{4}(350$ $\mu \mathrm{M})$. Los ingredientes declarados en el producto comercial a base de salvado de trigo BF son: trigo integral (59\%), salvado de trigo (18\%), harina de trigo integral, azúcar, extracto de malta de cebada, jarabe de glucosa, sal, aroma natural. Todos los medios fueron esterilizados en autoclave a $121^{\circ} \mathrm{C}$, a 15 lb por 15 min. Posteriormente, fueron inoculados con tres cilindros de $0.5 \mathrm{~cm}$ de diámetro, tomados de la periferia de cultivos con 5 días de crecimiento. La incubación fue realizada en un agitador orbital con control de temperatura (New Brunswick Scientific Co. G 25 Inc., Edison, NJ, USA) a $28^{\circ} \mathrm{C}$ y $150 \mathrm{rpm}$. Alícuotas de $2 \mathrm{~mL}$ fueron tomadas cada tercer día por un periodo de 30 días, las cuales fueron almacenadas a $-20{ }^{\circ} \mathrm{C}$ hasta el momento de su análisis. 


\section{Potenciales hemicelulolítico y amilolítico}

La evaluación de los potenciales hemicelulolítico y amilolítico fue realizada en medio sólido, utilizando los medios descritos por Sin et al. (2002). El medio base consistió en $0.1 \%$ de peptona, $0.01 \%$ de extracto de levadura y $1.6 \%$ de agar, suplementado con carboximetilcelulosa (2\%), xilano (2\%) o almidón (2\%) para determinar la actividad de celulasas, xilanasas y amilasas, respectivamente. Los medios fueron esterilizados en una autoclave a $121^{\circ} \mathrm{C}$ y $15 \mathrm{lb}$ por $15 \mathrm{~min}$. Una vez vertidos en las placas Petri, fueron inoculados en el centro con un cilindro de $0.5 \mathrm{~cm}$ de diámetro tomado de la periferia de cultivos con 5 días de crecimiento. Los medios fueron incubados a $28{ }^{\circ} \mathrm{C}$. El diámetro del crecimiento fue medido diariamente por tres días con un vernier. Para determinar el diámetro de hidrólisis enzimática fue utilizada una solución de yodo-yoduro. Todos los ensayos fueron realizados por triplicado.

El índice de degradación (ID) fue estimado con la siguiente fórmula:

$$
I D=\frac{D}{C}
$$

Donde: $\mathrm{D}$ es el diámetro de degradación y $\mathrm{C}$ es el diámetro de crecimiento.

\section{Obtención de concentrados enzimáticos}

Una vez definidas las cinéticas de producción de lacasa y seleccionado el medio de mayor producción, fueron preparados matraces de $2 \mathrm{I}$, conteniendo $1 \mathrm{I}$ del medio seleccionado, previamente esterilizado e inoculado con seis cilindros de $0.5 \mathrm{~cm}$ de diámetro tomado de la periferia de cultivos con 5 días de crecimiento. Los cultivos fueron incubados en un agitador orbital con control de temperatura (New Brunswick Scientific Co. G 25 Inc., Edison, NJ, USA) a $28^{\circ} \mathrm{C}$ y 150 rpm. A los 18 días de crecimiento, los sobrenadantes fueron recuperados por filtración y posteriormente concentrados con un cartucho de ultrafiltración con corte molecular de $10 \mathrm{kDa}$ en una membrana tipo espiral prep/scale TFF-6 PLCC (Merck Millipore, Germany). Después de ser recuperados los concentrados enzimáticos, fueron determinados los títulos de lacasa, celulasas, xilanasas y amilasas. Finalmente, fueron almacenados en congelación a $-20^{\circ} \mathrm{C}$ hasta su uso.

\section{Análisis estadístico}

La significancia estadística en la actividad enzimática en medio sólido y líquido fue determinada mediante un análisis de varianza ANOVA y posterior comparación de medias con una prueba Tukey $(\mathrm{P}<0.05)$. El análisis estadístico se llevó a cabo con el programa estadístico computacional InfoStat Versión 2020e.

\section{RESULTADOS Y DISCUSIÓN}

\section{Cinética de producción de lacasa}

La cinética de producción para la cepa LE90 mostró la mayor producción en el medio Tx2, seguido por Tx3 (Figura $1 \mathrm{~A})$. En ambos medios se observó un incremento en la
Tabla 1. Análisis de varianza de la producción de lacasa en diferentes medios.

Table 1. Analysis of variance of laccase production in different media.

\begin{tabular}{lcc}
\hline F.V. & G.L. & C.M. \\
\hline Rep & 2 & $2169.18 \mathrm{~ns}$ \\
Cepa & 2 & $24465.25^{*}$ \\
Medio & 2 & $11401853.68^{* *}$ \\
Error & 74 & 6905.18 \\
C.V. & & 12.76 \\
\hline
\end{tabular}

F.V.= fuente de variación; G.L.= grados de libertad; C.M.= cuadrados medios; Rep $=$ repetición; C.V. $=$ coeficiente de variación. ${ }^{* *}, \mathrm{P}<0.01 .{ }^{*}, \mathrm{P}<0.05 . \mathrm{ns}$, $P>0.05$.

A

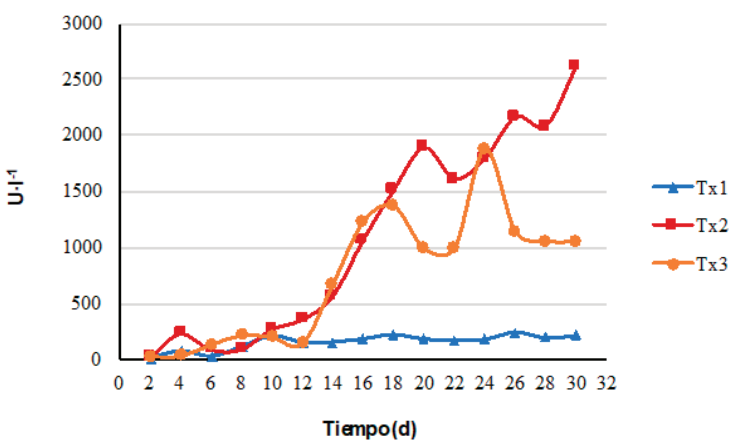

B

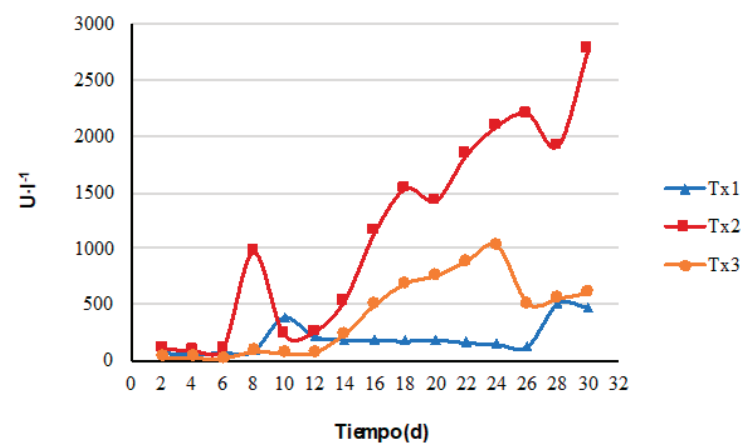

C

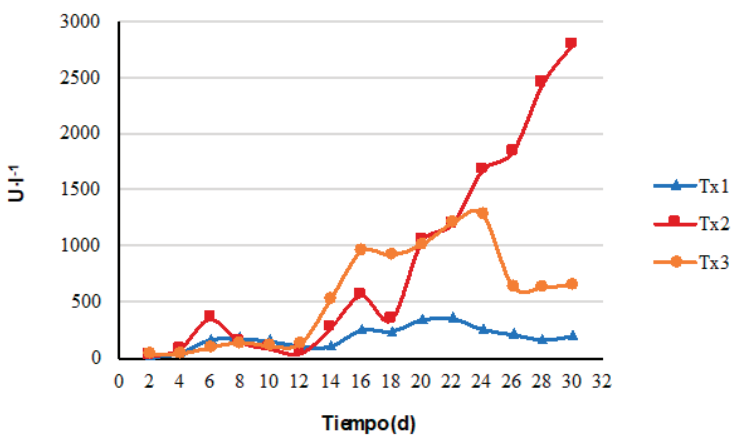

Figura 1. Curvas de producción de lacasa por Pycnoporus sangineus. Donde A) corresponde a la cepa LE90, B) LE133 y C) CH116 cultivadas en los tres medios de cultivo.

Figure 1. Laccase production curves by Pycnoporus sangineus. Where A) corresponds to strain LE90, B) LE133 and C) CH116 grew in the three-culture media. 
producción a partir del día 14, con picos máximos los días $20\left(1888.72 \mathrm{U} \cdot \mathrm{I}^{-1}\right), 26\left(2161.62 \mathrm{U} \cdot \mathrm{I}^{-1}\right)$ y $30\left(2617.78 \mathrm{U} \cdot \mathrm{I}^{-1}\right)$ para Tx2 y los días 18 (1367.59 U.l-1 ) y 24 (1876.49 U. - $\left.^{-1}\right)$ para Tx3. El medio Tx 1 fue el de menor producción, con títulos menores a $400 \mathrm{U} \cdot \mathrm{I}^{-1}$.

Las curvas de producción de lacasa para la cepa LE133 (Figura 1B) mostraron la mayor actividad en Tx2, donde la actividad incrementó sus títulos a partir del día 18 manteniendo el incremento a lo largo del tiempo hasta alcanzar 2792.36 U. $\mathrm{I}^{-1}$ al día 30. En el medio Tx3, la mayor producción fue observada entre los días 14 y 24, disminuyendo a partir del día 26. En el medio Tx1, LE133 mostró el mismo comportamiento que la cepa LE90, pero con menores títulos de producción.

En la Figura $1 \mathrm{C}$ se muestran las curvas de producción de la cepa $\mathrm{CH} 116$, que presentó los mayores títulos de producción en el medio Tx2, con un comportamiento ascendente a partir del día 14 y con picos máximos al día 16

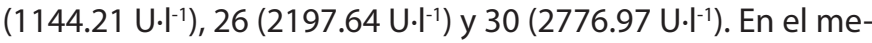
dio Tx1 fueron registrados los menores títulos de actividad. A diferencia de las cepas LE90 y LE133, CH116 mostró títulos inferiores a $1000 \mathrm{U} \cdot \mathrm{I}^{-1}$ en el medio Tx3.

En general, las tres cepas alcanzaron la mayor producción de lacasa en el medio Tx2. LE90 y CH116 mostraron un incremento exponencial a partir día 12 hasta el día 16. Sin embargo, existieron diferencias estadísticamente significativas entre las cepas $(P \leq 0.05)$ y los medios utilizados $(P \leq 0.01)$. En la tabla 2 son mostrados los resultados de la producción de lacasa en el medio Tx2, donde se comparan los títulos de las tres cepas en los días con mayor actividad, que podría estar asociado a la producción de diferentes isoformas.

Hay que destacar que Pycnoporus sanguineus es un género de basidiomicetos productor de lacasas termoestables (Orlikowska et al., 2018) con alto potencial redox (Zimbardi et al., 2016), considerando que la composición del medio influye en los títulos de producción (Zimbardi et al., 2016). Lo que explica, que las cepas estudiadas en el presente trabajo tuvieron la mayor actividad en el medio de cultivo Tx2. Además, éste podría ser el resultado de la presencia de inductores naturales como los componentes de pared celular del salvado de trigo (Riegas-Villalobos et al., 2020) y al cobre suplementado como inductor inorgánico (Zhuo et al., 2017). Sin embargo, los títulos fueron menores a lo reportado para la cepa $P$. sanguineus CS43 en condiciones similares de cultivo (Ramírez-Cavazos et al., 2014), pero similares a los observados en la cepa Pycnoporus sanguineus CS2 (GutiérrezSoto et al., 2015). Estas diferencias entre cepas pueden ser explicadas por la presencia de glucosa como fuente principal de carbono en combinación con otros inductores.

Por otro lado, la producción tuvo un comportamiento ascendente no lineal, cuyos picos podrían indicar la presencia de isoformas de lacasas, principalmente en los medios suplementados con cobre (Pezzella et al., 2012; Vrsanska et al., 2016). En este sentido, generalmente las cepas de Pycnoporus sanguineus producen al menos dos isoformas de lacasa (Li et al., 2016; Salazar-López et al., 2017; González-Bautista, 2019). La diversidad en las isoformas producidas por los hongos
Tabla 2. Actividad lacasa de cepas Pycnoporus sangineus en el medio de cultivo Tx2.

Table 2. Laccase activity of Pycnoporus sangineus strains in Tx2 culture medium.

\begin{tabular}{cccc}
\hline Día & \multicolumn{3}{c}{ Cepa } \\
& LE90 $\left(\mathbf{U}^{-1}\right)$ & LE133 $\left(\left.\mathbf{U}^{-1}\right|^{-1}\right)$ & CH116 $\left.\left(\mathbf{U}^{-1}\right)^{-1}\right)$ \\
\hline 10 & $277.13 \pm 16.99 \mathrm{a}$ & $87.83 \pm 8.97 \mathrm{c}$ & $228.48 \pm 10.79 \mathrm{~b}$ \\
\hline 16 & $1071.37 \pm 75.02 \mathrm{a}$ & $558.57 \pm 3.27 \mathrm{~b}$ & $1144.21 \pm 6.47 \mathrm{a}$ \\
\hline 18 & $1511.53 \pm 53.95 \mathrm{a}$ & $343.32 \pm 37.38 \mathrm{~b}$ & $1527.39 \pm 24.92 \mathrm{a}$ \\
\hline 20 & $1888.72 \pm 35.16 \mathrm{a}$ & $1036.49 \pm 8.62 \mathrm{c}$ & $1420.81 \pm 23.42 \mathrm{~b}$ \\
\hline 26 & $2161.62 \pm 10.4 \mathrm{a}$ & $1834.69 \pm 15.94 \mathrm{~b}$ & $2197.64 \pm 22.22 \mathrm{a}$ \\
\hline 30 & $2617.78 \pm 42.19 \mathrm{a}$ & $2792.36 \pm 62.56 \mathrm{a}$ & $2776.97 \pm 73.16 \mathrm{a}$ \\
\hline
\end{tabular}

Tx2: medio con All Bran Flakes ${ }^{\circledast}$ (BF) al $2 \%$, suplementado con CuSO (350 $_{4}$ $\mu \mathrm{M})$; LE90, LE133 y CH116: cepas de Pycnoporus sangineus nativas del estado de Nuevo León. Las medias con diferentes letras minúsculas dentro de la misma fila representan diferencia estadísticamente significativa (Tukey, $\mathrm{P}<0.05)$.

de esta especie sugiere una mayor capacidad para acceder a diferentes fuentes de carbono y nitrógeno, lo cual le concede un poder de adaptación en disímiles ambientes de crecimiento (Bertrand et al., 2015). El análisis de las isoformas constitutivas e inducidas de las cepas será abordado en otros estudios.

\section{Potenciales hemicelulolítico y amilolítico}

En la Figura 2 se presentan los resultados de los potenciales hemicelulolítico y amilolítico. La producción de amilasas no mostró diferencia estadísticamente significativa entre las cepas $(P>0.05)$, mientras que las celulasas y xilansas si mostraron diferencias $(\mathrm{P} \leq 0.05)$. Las cepas LE133 y CH116 tuvieron valores del ID de 1.2 para la celulosa. En cambio, $\mathrm{CH} 116$ mostró mayor actividad de xilanasas con índices de degradación mayores a 1.0. De forma general, las tres cepas mostraron mayor actividad de celulolítica concordando con lo reportado en aislados nativos de Nuevo León por MedinaGonzález et al. (2016). Sin embargo, los ID de las cepas de $P$. sanguineus fueron menores al rango mencionado por estos mismos autores de 1.032-2.559 y 1.077-1.188 para celulosa

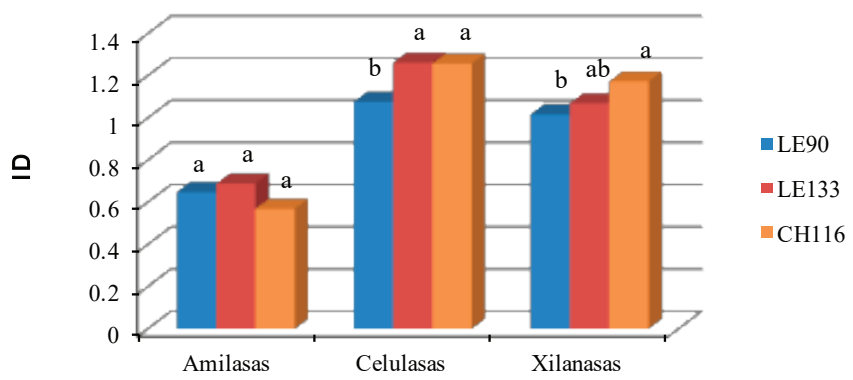

Figura 2. Índice de degradación enzimática (ID) de las cepas Pycnoporus sangineus. Las medias con diferentes letras minúsculas dentro de la misma clasificación representan diferencia estadísticamente significativa (Tukey, P $<0.05)$.

Figure 2. Pycnoporus sangineus strains enzyme degradation index (ID). The means with different lowercase letters within the same classification represent a statistically significant difference (Tukey, $\mathrm{P}<0.05$ ). 
y xilano, respectivamente. Este comportamiento puede ser explicado a nivel de secretoma para esta especie que, bajo condiciones de cultivo ricas en lignocelulosa, el $2 \%$ de las enzimas producidas por este hongo corresponden a las de actividad celulolítica y otro $2 \%$ a las hemicelulolíticas, mientras que las enzimas lignolíticas comprenden alrededor del $9 \%$ (Gauna et al., 2020).

Independientemente de los bajos niveles de celulasas y xilanasas es necesario un estudio de las condiciones óptimas de producción, ya que otras investigaciones han demostrado que estás actividades responden positivamente a la presencia de sustratos ricos en lignocelulosa (Rohr et al., 2013), sugiriendo bajos costos de producción enzimática.

Con respecto al potencial amilolítico, las cepas presentaron índices de degradación entre 0.57 y 0.69 , menores a los reportados para Aspergillus, Penicillium y Trichoderma por Khokhar et al. (2011). Sin embargo, cabe mencionar que las enzimas producidas por cepas Pycnoporus sanguineus han mostrado termoestabilidad (Gutiérrez-Soto et al., 2015; Orlikowska et al., 2018), lo que las hace atractivas para su aplicación a nivel industrial.

\section{Consumo de azúcares reductores}

Una vez identificadas las actividades de amilasas, celulasas y xilanasas en medio sólido, se determinó el comportamiento del consumo de azúcares en medio líquido, asociado a la producción de amilasas y hemicelulasas.

En la tabla 3 es mostrado el análisis de varianza, donde se observa diferencia estadísticamente significativa $(P \leq 0.05)$ entre el comportamiento de las cepas y los días de estudios, con interacción entre estos. En la figura 3 se presenta el consumo de los azúcares de las cepas, donde en los primeros ocho días se observó un alto consumo (aproximadamente el $55 \%$ ). Entre los días 10 y 18 la concentración disminuye cerca del $80 \%$, manteniendo un comportamiento de lento consumo, sugiriendo que los productos del metabolismo secundario aparecerán después del día 14 y que coincide con el incremento de la actividad de lacasa (Figura 1). Además, las fluctuaciones en la concentración de azúcares observadas los días 10 y 20 pueden estar asociadas a la presencia de enzimas que son inducibles (Álvarez et al., 2016). Cabe mencionar que el comportamiento observado entre los días 10 y 18 sugiere el término del metabolismo primario y el comienzo del metabolismo secundario.

Por otro lado, cabe mencionar que los basidiomicetos de la pudrición blanca se caracterizan por su potencial lignolítico (Riegas-Villalobos et al., 2020), además de producir amilasas, xilanasas y celulasas como parte de su metabolismo primario en presencia de almidón y componentes de pared celular (Kobakhidze et al., 2016; Naidu et al., 2016). Por lo que, al agotarse los azúcares simples presentes en el medio, serán expresadas las enzimas implicadas en la degradación de estos biopolímeros (Valadares et al., 2019; Okal et al., 2020). Esto puede observase en la cinética del consumo de azúcares en el medio Tx2 (BF), un medio complejo compuesto por trigo integral, salvado de trigo y harina de trigo, que lo hacen
Tabla 3. Análisis de varianza del consumo de azúcares reductores. Table 3. Analysis of variance of reducing sugar consumption

\begin{tabular}{lcc}
\hline F.V. & G.L. & C.M. \\
\hline Día & 11 & $0.21^{* *}$ \\
Rep & 3 & $0.01^{* *}$ \\
Día*Rep & 33 & 0.0017 \\
Cepa & 2 & $0.01^{* *}$ \\
Cepa*Día & 22 & $0.0032^{* *}$ \\
Cepa*Rep & 6 & $0.00019^{* *}$ \\
Error & 66 & 0.00057 \\
C.V. & & 11.21 \\
\hline
\end{tabular}

F.V.= fuente de variación; G.L.= grados de libertad; C.M.= cuadrados medios; Rep $=$ repetición; C.V. $=$ coeficiente de variación. ${ }^{* *}, \mathrm{P}<0.01$.

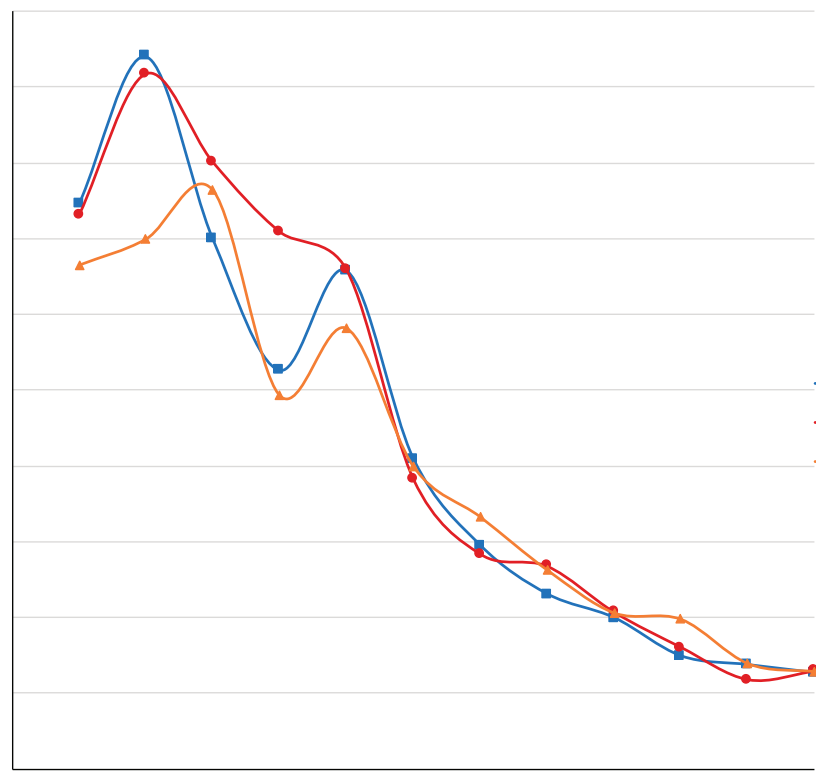

Figura 3. Consumo de azúcares por cepas Pycnoporus sangineus.

Figure 3. Reducing sugar consumption by Pycnoporus sangineus strains.

rico en almidón y hemicelulosa (Gómez et al., 2020). Además, el cereal esta adicionado con otros carbohidratos como sacarosa, extracto de malta de cebada y jarabe de glucosa que contribuyen al contenido de carbono.

\section{Obtención de concentrados enzimáticos}

En la Tabla 2 son mostrados los títulos de actividad de amilasas, celulasas, xilanasas y lacasa presentes en los concentrados obtenidos de los cultivos al día 18. La principal actividad producida por todas las cepas fue la de lacasa. EI cóctel obtenido a partir de la cepa $\mathrm{CH} 116$ tuvo los mayores títulos de lacasa (4500 $\left.\mathrm{U} \cdot \mathrm{I}^{-1}\right)$ y amilasa $\left(485 \mathrm{U} \cdot \mathrm{I}^{-1}\right)$, mientras que LE90 mostró la mayor concentración de xilanasas $\left(785 \mathrm{U} \cdot \mathrm{I}^{-1}\right)$. El concentrado LE133 fue el de mayor actividad de celulasa.

Por otra parte, los concentrados enzimáticos mostraron un patrón común de producción de enzimas, siendo la lacasa la más abundante, seguida de las xilanasas, amilasas y finalmente las celulasas. Este comportamiento concuerda con lo reportado para este género (Gauna et al., 2020). Por lo que, los bajos niveles observados podrían incrementarse mediante combinaciones de fuentes de carbono y sustratos 
Tabla 4. Actividad de los concentrados de cepas nativas de Pycnoporus sanguíneos.

Table 4. Enzyme concentrates activity of native Pycnoporus sangineus strains.

\begin{tabular}{lccc}
\hline \multirow{2}{*}{ Enzimas } & \multicolumn{3}{c}{ Cepa } \\
\cline { 2 - 4 } & LE90 $\left(\mathbf{U}^{-1}\right)$ & LE133 $\left(\left.\mathbf{U}^{-1}\right|^{-1}\right)$ & CH116 $\left(\mathbf{U}^{-1} \mathbf{- 1}^{-1}\right)$ \\
\hline Xilanasas & 785 & 328 & 128 \\
Celulasas & 0.0 & 271 & 120 \\
Amilasas & 86 & 29 & 485 \\
Lacasas & 3300 & 2100 & 4500 \\
\hline
\end{tabular}

LE90, LE133 y CH116: cepas de Pycnoporus sangineus nativas del estado de Nuevo León

ricos en lignocelulosa (Rohr et al., 2013; Kumla et al., 2020). Cabe mencionar que, esta combinación de lacasa-xilanasa estaría relacionada con la estrategia de descomposición de la lignocelulosa de estas cepas en su sustrato natural (Veloz Villavicencio et al., 2020). Esta combinación es de gran interés en el mejoramiento de las propiedades funcionales de las masas y la calidad final de sus productos (Niño-Medina et al., 2017). Con respecto a la combinación de a-amilasa, xilanasa y celulasa se ha demostrado su efecto sinérgico sobre las propiedades reológicas de la masa al reducir la absorción del agua $y$, por ende, en la extensibilidad, ablandamiento y el índice de tolerancia de mezclado de éstas (Liu et al., 2017). Sin embargo, estas enzimas aplicadas fueron obtenidas de diferentes casas comerciales. Por lo que, la obtención de concentrados enzimáticos a partir de un mismo organismo, creciendo en un medio de producción de bajo costo (como los residuos agroindustriales), de fácil recuperación y con las ventajas de termoestabilidad y rangos amplios de $\mathrm{pH}$, son algunos de los atributos que podrían hacer de estas cepas de Pycnoporus sanguineus excelentes candidatos para su aplicación a nivel industrial (Okal et al., 2020). Los aislados de este género son considerados organismos robustos, capaces de tolerar condiciones extremas de $\mathrm{pH}$ y temperatura. La termoestabilidad reportada en los sistemas lignocelulolíticos de cepas $P$. sanguineus demuestran su potencial aplicación en los procesos biotecnológicos (Gutiérrez-Soto et al., 2015; Orlikowska et al., 2018; Niderhaus et al., 2018). Por tal razón, serán requeridos futuros estudios para la optimización de la producción, así como de la caracterización de las propiedades operativas y funcionales de las enzimas producidas por estos aislados.

\section{CONCLUSIONES}

Gracias a la plasticidad metabólica de estos organismos, las enzimas expresadas en esta investigación pueden producirse en las cantidades aquí descritas o el medio puede ser modificado para obtener una en particular. Las cepas Pycnoporus sanguineus LE133 y $\mathrm{CH} 116$ tienen potencial para producir lacasa, celulasas, xilanasas y amilasas en un medio de bajo costo. Además, el cóctel enzimático de $P$. sanguineus LE133 puede ser aplicado en la industria de la panificación como mejorador de productos elaborados a base de harina integral.

\section{REFERENCIAS}

Abadulla, E., Tzanov, T., Costa, S., Robra, K.H., Cavaco-Paulo, A. y Gübitz, G.M. 2000. Decolorization and detoxification of textile dyes with a laccase from Trametes hirsuta. Applied and environmental microbiology. 66(8): 3357-3362.

Álvarez, J., Sánchez, C., Díaz, R. y Díaz-Godínez, G. 2016. Characterization of production of laccases, cellulases and xylanases of Pleurotus ostreaus grown on solid-state fermentation using an inert support. Revista Mexicana de Ingeniería Química. 15(2): 323-331.

Bertrand, B., Martínez-Morales, F., Tinoco-Valencia, R., Rojas, S., Acosta-Urdapilleta, L. y Trejo-Hernández, M.R. 2015. Biochemical and molecular characterization of laccase isoforms produced by the white-rot fungus Trametes versicolor under submerged culture conditions. Journal of Molecular Catalysis B: Enzymatic. 122: 339-347.

Drozlowska, E. 2019. The use of enzymatic fungal activity in the food industry-review. World Scientific News. 116: 222-229.

Gauna, A., Larran, A.S., Feldman, S.R., Permingeat, H.R. y Perotti, V. E. 2020. Secretome characterization of the lignocellulosedegrading fungi Pycnoporus sanguineus and Ganoderma applanatum. bioRxiv. Woodbury, NY

Gómez, M., Gutkoski, L.C.y Bravo-Núñez, Á. 2020. Understanding whole-wheat flour and its effect in breads: A review. Comprehensive Reviews in Food Science and Food Safety. 19(6): 3241-3265.

Gutiérrez -Soto, G., Medina-González, G.E., García- Zambrano, E.A., Treviño-Ramírez, J. E. y Hernández-Luna, E. 2015. Selection and Characterization of a Native Pycnoporus sanguineus Strain as a Lignocellulosic Extract Producer from Submerged Cultures of Various Agroindustrial Wastes. BioResources. 10(2): 3564-3576.

Kathirgamanathan, M., Abayasekara, C.L., Kulasooriya, S.A., Wanigasekera, A. y Ratnayake, R.R. 2017. Evaluation of 18 isolates of basidiomycetes for lignocellulose degrading enzymes. Ceylon Journal of Science. 46(4): 77-84.

Khokhar, I., Mukhtar, I. y Mushtaq, S. 2011. Isolation and screening of amylolytic filamentous fungi. Journal of Applied Sciences and Environmental Management. 15(1): 203-206.

Kobakhidze, A., Asatiani, M., Kachlishvili, E. y Elisashvili, V. 2016. Induction and catabolite repression of cellulase and xylanase synthesis in the selected white-rot basidiomycetes. Annals of Agrarian Science. 14(3): 169-176.

Kumla, J., Suwannarach, N., Sujarit, K., Penkhrue, W., Kakumyan, P., Jatuwong, K., Vadthanarat, S. y Lumyong, S. 2020. Cultivation of Mushrooms and Their Lignocellulolytic Enzyme Production Through the Utilization of AgroIndustrial Waste. Molecules. 25(12): 2811.

Li, S., Tang, B., Liu, Y., Chen, A., Tang, W. y Wei, S. 2016. Highlevel production and characterization of laccase from a newly isolated fungus Trametes sp. LS-10C. Biocatalysis and Agricultural Biotechnology. 8: 278-285.

Liu, W., Brennan, M. A., Serventi, L. y Brennan, C.S. 2017. Effect of cellulase, xylanase and a-amylase combinations on the rheological properties of Chinese steamed bread dough enriched in wheat bran. Food chemistry 234: 93-102. 
Medina-González, G.E., H. Bernal Barragán, C.E. Hernández-Luna, C.A. Hernández-Martínez, G. y Gutiérrez-Soto, G. 2016. Uso de basidiomicetos nativos en la biotransformación del pasto buffel (Cenchrus ciliaris) para mejorar la calidad nutricional. Revista Mexicana de Micología. 43: 31-35.

Miguel, Â.S.M., Martins-Meyer, T.S., Figueiredo, E.V.D.C., Lobo, B.W.P. y Dellamora-Ortiz, G.M. 2013. Enzymes in bakery: current and future trends. En: Food industry (Ed.), pp 278321. IntechOpen. Inc., Londres.

Miller, G.L. 1959. Use of dinitrosalicylic acid reagent for determination of reducing sugar. Analytical chemistry. 31(3): 426-428.

Naidu, Y., Siddiqui, Y. y Idris, A.S. 2020. Comprehensive studies on optimization of ligno-hemicellulolytic enzymes by indigenous white rot hymenomycetes under solid-state cultivation using agro-industrial wastes. Journal of environmental management. 259: 110056.

Niderhaus, C., Garrido, M., Insani, M., Campos, E., y Wirth, S. 2018. Heterologous production and characterization of a thermostable GH10 family endo-xylanase from Pycnoporus sanguineus BAFC 2126. Process Biochemistry. 67: 92-98.

Niño-Medina, G., Gutiérrez-Soto, G., Urías-Orona, V.y HernándezLuna, C.E. 2017. Effect of laccase from Trametes maxima CU1 on physicochemical quality of bread. Cogent Food \& Agriculture. 3(1): 1328762.

Okal, E.J., Aslam, M.M., Karanja, J.K. y Nyimbo, W.J. 2020. Mini review: Advances in understanding regulation of cellulase enzyme in white-rot basidiomycetes. Microbial Pathogenesis. 147: 104410.

Orlikowska, M., Rostro-Alanis, M.D.J., Bujacz, A., Hernández-Luna, C., Rubio, R., Parra, R. y Bujacz, G. 2018. Structural studies of two thermostable laccases from the white-rot fungus Pycnoporus sanguineus. International journal of biological macromolecules. 107: 1629-1640.

Patel, A.K., Singhania, R.R. y Pandey, A. 2016. Novel enzymatic processes applied to the food industry. Current Opinion in Food Science. 7: 64-72.

Patel, A.K., Singhania, R.R., y Pandey, A. 2017. Production, purification, and application of microbial enzymes. En: Biotechnology of Microbial Enzymes (ed.), pp. 13-41. Academic Press. Inc., Cambridge.

Pezzella, C., Lettera, V., Piscitelli, A., Giardina, P. y Sannia, G. 2012. Transcriptional analysis of Pleurotus ostreatus laccase genes. Applied Microbiology and Biotechnology. 97(2): 705-717.

Pozdnyakova, N.N., Turkovskaya, O.V., Yudina, E.N. y RodakiewiczNowak, Y. 2006. Yellow laccase from the fungus Pleurotus ostreatus D1: purification and characterization. Applied Biochemistry and microbiology. 42(1): 56-61.

Ramírez-Cavazos, L. I., Junghanns, C., Ornelas-Soto, N., CárdenasChávez, D.L., Hernández-Luna, C., Demarche, P., Enaud, E., García-Morales, R., Agathos, S.N. y Parra, R. 2014. Purification and characterization of two thermostable laccases from Pycnoporus sanguineus and potential role in degradation of endocrine disrupting chemicals. Journal of Molecular Catalysis B: Enzymatic. 108: 32-42.
Riegas-Villalobos, A., Martínez-Morales, F., Tinoco-Valencia, R., Serrano-Carreón, L., Bertrand, B. y Trejo-Hernández, M.R. 2020. Efficient removal of azo-dye Orange II by fungal biomass absorption and laccase enzymatic treatment. 3 Biotech. 10(4): 1-10.

Rohr, C.O., Levin, L.N., Mentaberry, A.N. y Wirth, S.A. 2013. A first insight into Pycnoporus sanguineus BAFC 2126 transcriptome. PloS one. 8(12): e81033.

Salazar-López, M., Rostro-Alanis, M.D.J., Castillo-Zacarías, C., Parra-Guardado, A.L., Hernández-Luna, C., lqbal, H.M. y ParraSaldivar, R. 2017. Induced degradation of anthraquinonebased dye by laccase produced from Pycnoporus sanguineus (CS43). Water, Air, \& Soil Pollution. 228(12): 1-10.

Sin, M.K., Hyde, K.D. y Pointing, S.B. 2002. Comparative enzyme production by fungi from diverse lignocellulosic substrates. Journal of Microbiology. 40(3): 241-244.

Singh, P. y Kumar, S. 2019. Microbial enzyme in food biotechnology. En: Enzymes in Food Biotechnology (ed.), pp 19-28. Academic Press. Inc., Cambridge.

Singh, R., Singh, A. y Sachan, S. 2019. Enzymes used in the food industry: Friends or foes? En: Enzymes in food biotechnology (ed.), pp 827-843. Academic Press. Inc., Cambridge.

Tebben, L., Shen, Y. y Li, Y. 2018. Improvers and functional ingredients in whole wheat bread: A review of their effects on dough properties and bread quality. Trends in Food Science \& Technology. 81: 10-24.

Valadares, F., Gonçalves, T.A., Damasio, A., Milagres, A.M., Squina, F.M., Segato, F. y Ferraz, A. 2019. The secretome of two representative lignocellulose-decay basidiomycetes growing on sugarcane bagasse solid-state cultures. Enzyme and microbial technology. 130: 109370.

Veloz Villavicencio, E., Mali, T., Mattila, H.K., y Lundell, T. 2020. Enzyme activity profiles produced on wood and straw by four fungi of different decay strategies. Microorganisms. 8(1): 73.

Vrsanska, M., Voberkova, S., Langer, V., Palovcikova, D., Moulick, A., Adam, V. y Kopel, P. 2016. Induction of Laccase, Lignin Peroxidase and Manganese Peroxidase Activities in WhiteRot Fungi Using Copper Complexes. Molecules. 21(11): 1553.

Wang, W., Liu, F., Jiang, Y., Wu, G., Guo, L., Chen, R., Chen, B., Lu, Y., Dai, Y. y Xie, B. 2015. The multigene family of fungal laccases and their expression in the white rot basidiomycete Flammulina velutipes. Gene. 563(2): 142-149.

Zhang, Y., He, S. y Simpson, B.K. 2018. Enzymes in food bioprocessing - novel food enzymes, applications, and related techniques. Current Opinion in Food Science. 19: 30-35.

Zhuo, R., Yuan, P., Yang, Y., Zhang, S., Ma, F. y Zhang, X. 2017. Induction of laccase by metal ions and aromatic compounds in Pleurotus ostreatus HAUCC 162 and decolorization of different synthetic dyes by the extracellular laccase. Biochemical Engineering Journal. 117: 62-72.

Zimbardi, A., Camargo, P., Carli, S., Aquino Neto, S., Meleiro, L., Rosa, J., Andrade, A., Jorge, J. y Furriel, R. 2016. A High Redox Potential Laccase from Pycnoporus sanguineus RP15: Potential Application for Dye Decolorization. International Journal of Molecular Science. 17(5): 672. 